\title{
Ductal dilatation and stenting for residual hepatolithiasis: a promising treatment strategy
}

\author{
S-M Sheen-Chen, Y-F Cheng, F-C Chen, F-F Chou, T-Y Lee
}

\begin{abstract}
Background and aims-Hepatolithiasis is prevalent in south-east Asia and presents a difficult management problem. Intrahepatic strictures with or without awkward ductal angulation of the biliary tree are the main reasons for the reported high incidence of postoperative residual stones. Without proper treatment, biliary strictures and residual stones can lead to repeated episodes of cholangitis, liver abscess, secondary biliary cirrhosis, portal hypertension, and death from sepsis or hepatic failure. The purposes of our treatment strategy were to achieve complete clearance of the stones and relief of bile stasis.
\end{abstract}

Methods-From January 1991 to July 1992, 90 patients with residual postoperative hepatolithiasis and intrahepatic strictures were treated. Postoperative ductal dilatation with percutaneous transhepatic cholangioscopy tube stenting through a mature $T$ tube tract was performed. Choledochoscopic electrohydraulic lithotripsy was applied when impacted or large stones were encountered.

Results-Complete clearance of stones was achieved in 78 patients $(87 \%)$. Mild haemobilia occurred in five patients $(5.5 \%)$ and fever developed in seven patients $(7.7 \%)$, and these patients recovered after conservative treatment. The rate of stone recurrence after a mean follow up of 43 months was $8 \%$. Intrahepatic cholangiocarcinoma developed in one patient (1.1\%).

Division of General Surgery, Department of Surgery and Diagnostic Radiology, Chang Gung Memorial Hospital, Kaohsiung, Chang Gung Medical College, Kaohsiung

Hsien, Taiwan

S-M Sheen-Chen

Y-F Cheng

F-C Chen

F-F Chou

T-Y Lee

Correspondence to: Dr S-M Sheen-Chen, Division of General Surgery, Department of Surgery, Chang Gung Memorial Hospital, Kaohsiung, Chang Gung Medical College, 123 Ta-Pei Road, Niao-Sung Hsiang, Kaohsiung Hsien, Taiwan.

Accepted for publication 9 October 1997
Conclusion-Postoperative ductal dilatation and stenting, combined with endoscopic electrohydraulic lithotripsy when indicated, is an effective and safe treatment with a low recurrence rate for complicated residual hepatolithiasis with biliary stricture.

(Gut 1998;42:708-710)

Keywords: hepatolithiasis; biliary stricture; electrohydraulic lithotripsy

Hepatolithiasis is prevalent in south-east Asia and is a difficult management problem. ${ }^{1-12}$ The main purposes of treatment are clearance of the stones and elimination of bile stasis. Many patients present with associated intrahepatic strictures, tortuous bile ducts, and large impacted stones. Consequently, failure of complete duct clearance following surgical intervention is reported to be as high as $77 \% .{ }^{1314}$ Without proper treatment, biliary strictures and residual stones can lead to repeated episodes of cholangitis, liver abscess, secondary biliary cirrhosis, portal hypertension, and death from sepsis or hepatic failure. ${ }^{15}$ Dilatation of the intrahepatic strictures and retrieval of the residual stones may prevent these complications. We have designed a clinical strategy of ductal dilatation through mature $\mathrm{T}$ tube tracts, percutaneous transhepatic cholangioscopy (PTCS) tube stenting, followed by choledochoscopic stone retrieval.

\section{Methods}

From January 1991 to July 199490 patients with residual postoperative hepatolithiasis and intrahepatic strictures were treated surgically. Of these 90 patients, 31 had had their gall bladder removed on a previous occasion and so underwent extended supraduodenal choledocholithotomy only. The other 59 patients underwent both cholecystectomy and extended supraduodenal choledocholithotomy. Intraoperative choledochoscopy was undertaken in all patients. As many stones as possible were removed during surgery, whilst calculi peripheral to strictures were left for extraction by subsequent $\mathrm{T}$ tube track ductal dilatation and postoperative choledochoscopy.

Dilatation of mature $\mathrm{T}$ tube tracks with a PTCS tube (Sumitomo, Tokyo, Japan) was undertaken at Chang Gung Memorial Hospital, Kaohsiung, Taiwan. Fifty two of the patients were women. Ages ranged from 28 to 81 years (mean 49.8 years). Forty six patients $(51 \%)$ had right intrahepatic strictures, 26 $(29 \%)$ had left intrahepatic strictures, and 18 $(20 \%)$ had bilateral intrahepatic strictures. Ductal dilatation was usually started four weeks after the operation. A guidewire was first introduced through the mature $\mathrm{T}$ tube track and passed through the intrahepatic strictures under fluoroscopic examination. Strictures were dilated with a Teflon dilator (Medi-Tech, Westwood, Massachusetts, USA). A PTCS tube (12 or $14 \mathrm{~F}$ ) was then used for stenting. The intrahepatic biliary strictures were dilated and stented gradually at intervals of one week until an 18-20 F tract was achieved. An improved fibreoptic choledochoscope (CHF P10, Olympus, Tokyo, Japan) was used after the removal of the PTCS tube, and a grasping basket was inserted through the instrument channel to remove the stones (fig 1). Electrohydraulic lithotripsy was used to disrupt impacted or large stones in 58 patients. The generator used was a Riwolith Impalso Pressure Generator 2137 (Richard Wolf GmbH, Knittliger, Germany). The electrohydraulic probe (No 5F) was kept in close proximity to the 


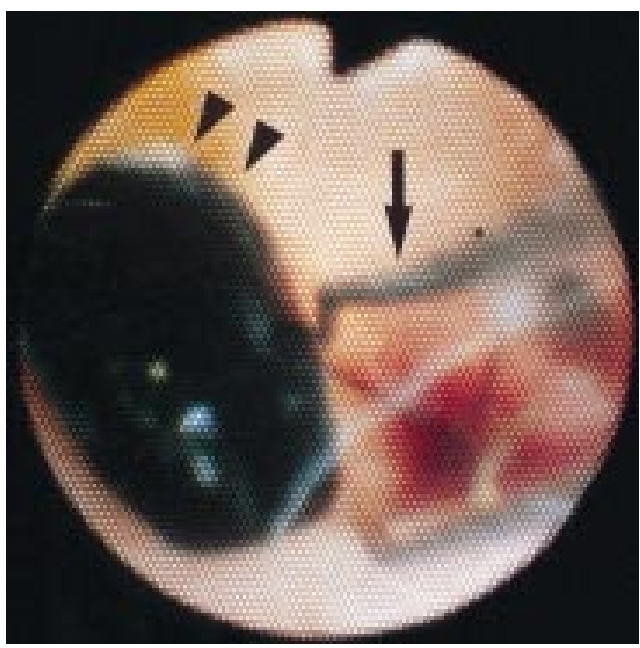

Figure 1 After ductal dilatation the stones (arrowheads) were clearly visualised. The basket (arrows) was then used to extract the calculi.

stone through the channel of the choledochoscope, and shock waves were applied until the stone fragmented. The whole procedure took approximately 20 minutes and was continuously monitored under direct vision through the choledochoscope. The fragmented stones were retrieved by saline irrigation and by the use of baskets. Choledochoscopy with stone retrieval was repeated at intervals of 1-2 weeks until complete clearance of stones was achieved. Electrohydraulic lithotripsy was also repeated if necessary. Intrahepatic ducts were stented with a suitably sized (18-20 F) PTCS tube during the treatment period and for at least three months after complete clearance of stones. Confirmation of clearance was verified by cholangioscopy and cholangiography. Only if cholangiography, performed after three months, showed no recurrence or ductal strictures was the tube removed.

Both ductal dilatation and cholangioscopic lithotripsy were performed as outpatient procedures. Most patients were able to tolerate this without medication although $50 \mathrm{mg}$ pethidine was administered intramuscularly to some

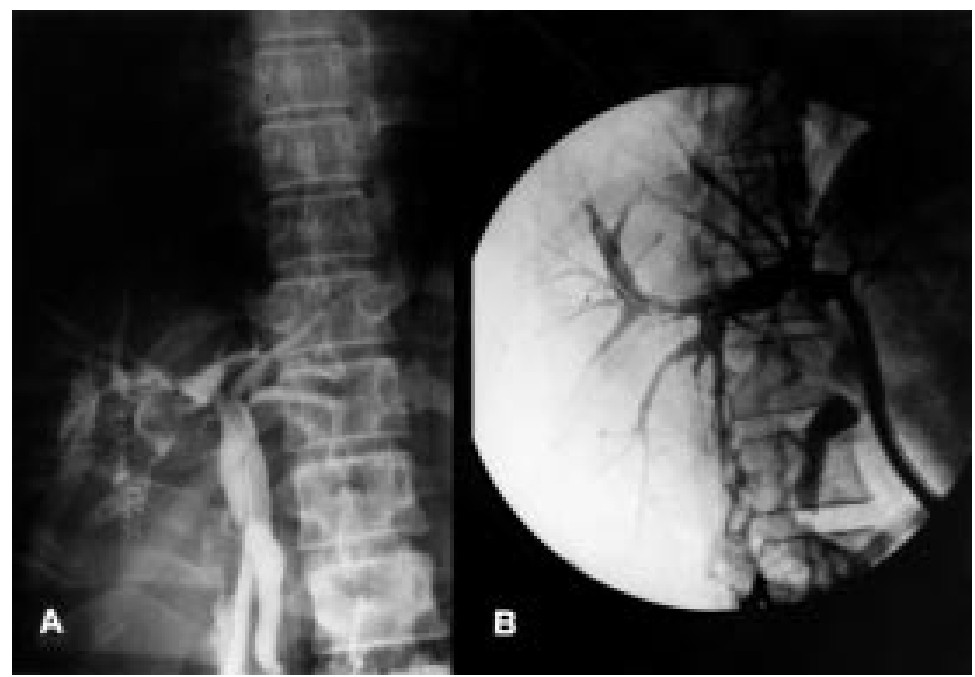

Figure 2 (A) Residual stones with PTCS tube stenting. (B) Complete clearance of stones was achieved and excellent visualisation of the biliary tree was shown by cholangiography. patients. During the period of stenting the patients were seen monthly in the outpatient clinic. The external tip of the tube was closed for most of the time and was occasionally released to check its patency. If any malfunction or displacement was suspected, a new tube was inserted or the tube was repositioned.

\section{Results}

Eighty patients underwent three ductal dilatations and stenting procedures and 10 patients needed two procedures. Four hundred and five choledochoscopies for the removal of stones were performed after ductal dilatation in the 90 patients (range $2-8$; median 5 per patient). The intrahepatic stones ranged in size from 0.4 to $3.2 \mathrm{~cm}$ (median $1.8 \mathrm{~cm}$ ). Eighty one endoscopic electrohydraulic lithotripsies were performed in 58 patients (range 1-3; median 1 per patient). Complete clearance of the stones was achieved in 78 patients (fig 2). The total number of procedures (ductal dilatation and stenting and choledochoscopic procedures) was $665(260+405)$. The number of procedures needed per patient ranged from 4 to 11 (median 8).

No mortality or major morbidity occurred. Mild haemobilia was noted in five patients $(5.5 \%)$ and fever developed in seven patients $(7.7 \%)$. All recovered after conservative treatment. The 78 successfully treated patients underwent abdominal ultrasonography at six month intervals. Seven patients $(8 \%)$ developed ultrasound evidence of stone recurrence after a mean follow up of 43 months (range 22-61 months). Intrahepatic cholangiocarcinoma developed in one patient with left intrahepatic structures after two years of follow up.

\section{Discussion}

The management of hepatolithiasis is based upon complete clearance of stones, elimination of biliary strictures, and prevention of stone recurrence. ${ }^{13}$ In spite of advances in choledochofibreoscopy, intrahepatic strictures, tortuously angled ducts, and the presence of large and impacted stones are the major obstacles to success. Retained calculi are common following common bile duct exploration because it is difficult or impossible to pass instruments through strictures associated with such stones. Forceful or blind instrument manipulation during surgery may create haemobilia or fistula without successful stone retrieval.

Dilatation and stenting was safely performed in our series without severe complication. Furthermore, electrohydraulic lithotripsy safely fragmented intrahepatic calculi. Others have also reported effective treatment of calculi via $\mathrm{T}$ tube tracks, ${ }^{16}$ endoscopic retrograde cholangiopancreatography (ERCP), ${ }^{17}$ and through percutaneous transhepatic tracks. ${ }^{18}$

ERCP and peroral choledochoscopy can be used to manage most patients who present with extrahepatic bile duct stones but this approach may fail in the presence of stones peripheral to intrahepatic strictures. ${ }^{17}$ Extracorporeal shock wave lithotripsy is also usually unsuccessful because of the strictures and associated stone impaction. ${ }^{4}$ We did not use laser lithotripsy in 
this series; previous reports suggest that laser lithotripsy is less satisfactory than electrohydraulic lithotripsy for the fragmentation of bile duct stones. ${ }^{19}$ Surgery alone without postoperative ductal dilatation is associated with a high incidence of retained calculi. ${ }^{13} 14$

Intrahepatic cholangiocarcinoma and hepatolithiasis co-exist in $2.36-10.0 \%$ of cases. $^{20}$ Although there is no conclusive evidence that stones cause the development of cholangiocarcinoma, bile stasis and bacterial infection have been claimed to be the most important causative factors. ${ }^{21} 22$ One of our cases developed cholangiocarcinoma in a left bile duct two years after clearance of stones and ductal dilatation.

Stenting after ductal dilatation is mandatory for hepatolithiasis with intrahepatic strictures to prevent restricture, bile stasis, and reformation of stones. There are very few reports of stenting for intrahepatic calculi. The optimum duration of stenting is unclear. We adopted stenting for at least three months after clearance of the stones and this was associated with a stone recurrence rate of $8 \%$ after a mean follow up of 43 months. Jeng et al recommended stenting for at least six months in a more complicated series of patients who had secondary cirrhosis, multiple intrahepatic long strictures, and a history of repeated biliary surgery. ${ }^{23}$

We conclude that postoperative ductal dilatation and stenting, combined with endoscopic electrohydraulic lithotripsy when indicated, is an effective and safe treatment with a low recurrence rate for managing complicated residual hepatolithiasis with intrahepatic strictures.

The authors wish to express their gratitude to Miss Chiu-Yun Huang and Chih-Chi Sheen for their preparation of the manuscript.

1 Sheen-Chen SM, Chou FF. Postoperative choledochoscopy: is routine antibiotic prophylaxis necessary? A prospective randomized study. Surgery 1994;115:170-5.
$2 \mathrm{Su} \mathrm{CH}$, Lui WY, Peng FK. Relative prevalence of gallstones disease in Taiwan: a nationwide cooperative study. Dig Dis Sci 1992;37:764-8.

3 Sheen-Chen SM, Cheng YF, Lee Tze-Yu. Postoperative T-tube cholangiography: is routine antibiotic prophylaxis necessary? A prospective controlled study. Arch Surg 1995; 130:20-3.

4 Ker CG, Hwang CH, Chen JS, et al. Extracorporeal shockwave lithotripsy for treatment of intrahepatic stones: in vitro and in vivo studies. Hepatogastroenterology 1993;40: vitro and $159-62$.

5 Sheen-Chen SM, Chou FF. Choledochotomy for biliary lithiasis: is routine T-tube drainage necessary? A prospective controlled trial. Acta Chir Scand 1990;156:387-90.

6 Lee WJ, Chang KJ, Lee CS, et al. Surgery in cholangitis: bacteriology and choice of antibiotic. Hepatogastroenterology 1992;39:347-9.

7 Liu TJ. Acute biliary septic shock. HPB Surg 1990;2:17783.

8 Sheen-Chen SM, Chou FF. Is sterile water irrigation safe for postoperative choledochoscopy? A prospective trial. Eur $\mathcal{F}$ Surg 1996;162:801-4.

9 Jeng KS, Shih SC, Chiang HJ, et al. Secondary biliary cirrhosis: a limiting factor in the treatment of hepatolithiasis. Arch Surg 1989;124:1301-5.

10 Sheen-Chen SM, Eng HL, Cheng YF, et al. Circulating intercellular adhesion molecule-1 (ICAM-1) in patients with hepatolithiasis. Dig Dis Sci 1996;41:1682-6.

11 Sheen-Chen SM, Eng HL, Chou WJ. Serum-souble interleukin-2 receptor in patients with hepatolithiasis: a preliminary report. Hepatogastroenterology (in press).

12 Sheen-Chen SM, Cheng YF, Chou FF, et al. Percutaneous transhepatic cholangioscopy and lithotripsy for complicated hepatolithiasis. Asian f Surg 1996;19:284-5.

13 Choi TK, Wong J, Ong GB. The surgical management of primary intra-hepatic stones. Br F Surg 1982;69:86-90.

14 Chang TM, Passaro E Jr. Intrahepatic stones: The Taiwan experience. Am f Surg 1983;146:241-4.

15 Nakanuma Y, Yamaguchi K, Ohta G, et al. Japanese hepatolithiasis study group: pathologic features of hepatolithiasis in Japan. Hum Pathol 1988;19:1181-6.

16 Burhenne JH. Electrohydraulic fragmentation of retained common bile duct stones. Radiology 1975;117:721-2.

17 Koch H, Stolte M, Walz V. Endoscopic lithotripsy in the common bile duct. Gastrointest Endosc 1980;26:16-18.

18 Mo LR, Hwang MH, Yeuh, et al. Percutaneous transhepatic choledochoscopic electrohydraulic lithotripsy (PTCSEHL) of common bile duct stones. Gastrointest Endosc 1988;34:122-5.

19 Chen MF, Jan YY. Percutaneous transhepatic cholangioscopic lithotripsy. Br f Surg 1990;77:530-2.

20 Sheen-Chen SM, Chou FF, Eng HL. Intrahepatic cholangiocarcinoma in hepatolithiasis: a frequently overlooked disease. F Surg Oncol 1991;47:131-5.

21 Koga A, Ichimiya H, Yamaguchi $\mathrm{K}$, et al. Hepato-lithiasis associated with cholangiocarcinoma: possible etiological significance. Cancer 1985;55:2826-9.

22 Falchuk KR, Lesser PB, Galdabini J, et al. Cholangiocarcinoma as related to chronic intrahepatic cholangitis and hepatolithiasis. Am f Gastroenterol 1976;66:57-61.

23 Jeng KS, Yang FS, Chiang HJ, et al. Bile duct stent in the management of hepatolithiasis with long-segment intrahepatic biliary strictures. Br F Surg 1992;79:663-6. 